\title{
Werkstattbericht über das Projekt \\ "Die Südtiroler SchülerInnen und die Zweitsprache: eine linguistische und sozialpsychologische Untersuchung"
}

Andrea Abel (Bozen)

\begin{abstract}
The aim of the project "South-Tyrolean pupils and their second language: a linguistic and socio-psychological investigation", which was originated in the collaboration between the EURAC and the University of Trento, is to analyze the second language competence of South-Tyrolean pupils and to contextualize the results of such an investigation by commenting on crucial sociolinguistic and psychosocial aspects that influence it. In this paper some preliminary results of the project's preparatory study, i.e. an interview study as well as focus groups, are presented.
\end{abstract}

\section{Projektbeschreibung und -ziele}

Das Gemeinschaftsprojekt "Die Südtiroler SchülerInnen und die Zweitsprache: eine linguistische und sozialpsychologische Untersuchung" zwischen dem Institut für Fachkommunikation und Mehrsprachigkeit der Europäischen Akademie Bozen (Projektgesamtleitung: Andrea Abel und Chiara Vettori) und dem Fachbereich für Kognitions- und Erziehungswissenschaften der Universität Trient (Maria Paola Paladino), entsteht in enger Zusammenarbeit mit dem Deutschen und Italienischen Schulamt der Autonomen Provinz Bozen (Rita Gelmi, Walter Cristofoletti) und den Südtiroler Oberschulen. Eine ganze Reihe von MitarbeiterInnen sind inhaltlich und organisatorisch in das Projekt eingebunden, das nicht nur die Rolle der Varietäten, sondern auch weitere Einflussfaktoren beim Zweitsprachlernen untersuchen möchte.

Ziele des Projekts sind einerseits eine umfassende Dokumentation und Analyse der Zweitsprachkompetenzen der Südtiroler SchülerInnen der Sekundarstufe II auf linguistischer und soziolinguistischer sowie sozialpsychologischer Ebene und andererseits die Untersuchung von Zusammenhängen zwischen sprachlicher Leistung und außersprachlichen Faktoren und die Ermittlung besonders solcher Faktoren, die sich auf Sprachkenntnisse und sprachliches Verhalten positiv oder negativ auswirken.

$\mathrm{Zu}$ den Arbeitsschwerpunkten des Projekts gehören daher zum einen eine (fehler-) linguistische Untersuchung schriftlicher und mündlicher Äußerungen von Südtiroler SchülerInnen der Sekundarstufe II und zum anderen eine Erhebung (mittels Interviews, Fokusgruppen und Fragebogen) von Faktoren, die Erlernung und Gebrauch der Zweitsprache der SchülerInnen beeinflussen.

Im vorliegenden Beitrag werden erste Ergebnisse der qualitativen Vorstudien, die mittels Interviews und Fokusgruppen durchgeführt wurden, vorgestellt. 


\subsection{Hintergrund}

Den Hintergrund der Studie bildet die sprachliche Situation in Südtirol, das seit $1919 \mathrm{zu}$ Italien gehört und wo auf einer Gesamtfläche von rund $7400 \mathrm{~km}^{2}$ rund 500.000 Einwohner vor allem deutscher, italienischer und ladinischer Muttersprache leben. Laut den Ergebnissen der letzten Volkszählung aus dem Jahr 2001 bildet die deutsche Sprachgruppe 69,38\% der Bevölkerung und lebt sowohl in den Städten als auch im ländlichen Bereich, während die italienische Sprachgruppe, die 26,30\% der Bevölkerung ausmacht, vorwiegend in den Städten zuhause ist. Die ladinische Sprachgruppe mit einem Anteil von 4,32\% an der Gesamtbevölkerung ist hauptsächlich in den beiden Dolomitentälern (Gröden und Gadertal) beheimatet. ${ }^{1}$

Was den Status der deutschen Sprache in Südtirol betrifft, so sind die wichtigsten Bestimmungen in Bezug auf die Sprachenrechte (wie etwa die Sprachengleichstellung, der Sprachgruppenproporz, die Sprachgruppenzugehörigkeitserklärung, die Zweisprachigkeitsprüfung) durch den Pariser Vertrag von 1946 und vor allem durch das Zweite Autonomiestatut von 1972 geregelt. Danach ist die "deutsche Sprache [...] in der Region der italienischen Sprache, die die amtliche Staatssprache ist, gleichgestellt [...]" (Art. 99 des Autonomiestatuts). "Die deutschsprachigen Bürger der Provinz Bozen haben das Recht, im Verkehr mit den Gerichtsämtern und mit den Organen und Ämtern der öffentlichen Verwaltung [...] ihre Sprache zu gebrauchen." (Art. 100 des Autonomiestatuts). "In der Provinz Bozen wird der Unterricht in den Kindergärten, Grund- und Sekundarschulen in der Muttersprache der Schüler, das heißt in italienischer oder deutscher Sprache, von Lehrkräften erteilt, für welche die betreffende Sprache ebenfalls Muttersprache ist. In den Grundschulen [...] und in den Sekundarschulen ist der Unterricht der zweiten Sprache Pflicht [...]" (Art. 19 des Autonomiestatuts) (aus: Das neue Autonomiestatut 2003).

Die Erlernung der beiden Sprachen Deutsch und Italienisch wird in Südtirol mit beträchtlichem Aufwand gefördert: Die Zweitsprache wird als Fach nicht nur ab der ersten Klasse Grundschule bis zum Ende der Schulzeit unterrichtet (mehr als 2000 Unterrichtsstunden von der Grundschule bis zur Matura), sondern es gibt eine Reihe von besonderen Maßnahmen zur Verbesserung der Sprachkenntnisse, wie die Förderung von Sprachkursen, die Unterstützung für Auslandsaufenthalte, Partnerschaften mit Schulen anderer Unterrichtssprache, eine zunehmende Anzahl von Schulversuchen mit erweitertem Deutschunterricht und Sachfachunterricht in der Zweitsprache besonders an Schulen mit italienischer Unterrichtssprache (cf. Abel 2007: 236-241, Abel/Stuflesser 2006: 65). Trotz des beachtlichen Einsatzes an Mitteln und der vielen Initiativen sind

die Zweitsprachkenntnisse nicht immer zufriedenstellend, wie verschiedene Untersuchungen über die Zweitsprachkompetenzen, Umfragen zur Selbsteinschätzung der eigenen Sprachkenntnisse oder die Ergebnisse der Zweisprachigkeitsprüfung ${ }^{2}$ zeigen (cf. Putzer/ Deflorian 1997, CENSIS 1997, Egger 2001, Vettori 2004, ASTAT 2006a, ASTAT 2006b). So haben beispielsweise im Jahr 2005 lediglich 45,9\% der KandidatInnen die Zweisprachigkeitsprüfung bestanden (ASTAT 2006b). Laut Südtiroler Sprachbarometer (ASTAT 2006a), der im Jahr 2004 mit eine repräsentativen Stichprobe der Südtiroler Bevölkerung (Erwachsene ab dem 19. Lebensjahr), durchgeführt wurde, gibt $40 \%$ der italienischsprachigen Bevölkerung an, nur einzelne Wörter auf (Hoch-)Deutsch sagen zu können, während 5,1\% der Deutsch-

\footnotetext{
1 Die besondere Situation der ladinisch-sprachigen Bevölkerung bildet keinen Schwerpunkt im Rahmen der vorgestellten Studie und wird daher in diesem Beitrag auch nicht weiter vertieft.

2 Die Zweisprachigkeitsprüfung dient zum Nachweis der Deutsch- und Italienischkenntnisse (Prüfungen mit 4 Schwierigkeitsgraden, die nach dem Gemeinsamen Europäischen Referenzrahmen von A1-B2 reichen). Sie bildet die durch das Autonomiestatut festgelegte Voraussetzung dafür, eine Stelle in der öffentlichen Verwaltung in der autonomen Provinz Bozen-Südtirol antreten zu können.
} 
"Die Südtiroler SchülerInnen und die Zweitsprache"

sprachigen dasselbe in Bezug auf das Italienische aussagt, ein Ergebnis, das die vielfach öffentlich vorgebrachten Vermutungen über die Zweitsprachkenntnisse der beiden Sprachgruppen zu bestätigen scheint.

Die tatsächliche Verwendung des Deutschen durch italienische Muttersprachler wird besonders durch das Spannungsfeld bzw. die Diglossiesituation zwischen den deutschen Dialekten und der Hochsprache beeinflusst (cf. ASTAT 2006a, Egger 2004).

Abel/Stuflesser 2006: 65f.

Dem "Südtiroler Sprachbarometer" (ASTAT 2006a) ist auch zu entnehmen, dass die Kenntnis des "deutschen Dialekts" nach Ansicht von 88,7\% der deutschsprachigen Bevölkerung für ein gutes Zusammenleben wichtig ist, während nur 62,9\% der italienischen Sprachgruppe diese Meinung teilt (ASTAT 2006a: 171). 40,1\% der italienischen Sprachgruppe nennt den häufigen Gebrauch des Dialekts, der nicht mit dem in der Schule erlernten Deutsch übereinstimmt, als eines der Haupthindernisse beim Zweitsprachlernen, gefolgt vom Schulsystem, das - strikt getrennt in Schulen mit deutscher und solchen mit italienischer Unterrichtssprache - den aktuellen Erfordernissen nicht mehr gerecht werde. Beide Sprachgruppen sind (mit rund 40\%) ziemlich einheitlich der Auffassung, dass schlichtweg mangelnder Wille das Sprachenlernen erschwert; unterschiedlich ist die Auffassung in Bezug auf das mangelnde Interesse als lernhemmender Faktor: 57,2\% bei der deutschen und 14,3\% bei der italienischen Sprachgruppe (ASTAT 2006a: 176f.).

Die mangelnden Kenntnisse der Zweitsprache sowie die z. T. festzustellende Distanz dazu steht zweifelsohne in einem Zusammenhang mit der - durch das nach Sprachen getrennte Schulsystem bedingten - vorwiegend einsprachigen und der aus historischer Perspektive durchaus erklärbaren Sozialisation der Jugendlichen, in der die Zweitsprache mehr den Stellenwert eines Schulfachs denn ein Instrument zur Kommunikation im Alltag besitzt (cf. Abel/Stuflesser im Druck); außerdem lässt sie sich durch die unterschiedliche Verteilung der Sprachgruppen auf dem Territorium erklären (cf. Abel/Stuflesser, in Druck, Lanthaler 2006: 373, Baur 2000: 68-73).

Darüber hinaus zeigt die Sprachkontaktsituation in Südtirol, dass physische Nähe, das heißt das Leben mehrerer Sprachgruppen in ein- und demselben Territorium, "paradoxerweise nicht unbedingt mit Dialogbereitschaft einhergeht. Das wird z. T. aus historischen Situationen erzwungener Nähe heraus erklärt (cf. Baur 2000: 31). Demnach bedeutet Nähe nicht automatisch, dass das Sprachenlernen dadurch leichter wird." (Abel/Stuflesser 2006: 66).

Das lässt unter anderem auch die Tatsache erkennen, dass Freundschaften hauptsächlich innerhalb der eigenen Sprachgruppe geschlossen werden. Das Privatleben verläuft großteils einsprachig, auf Deutsch oder Italienisch. Die Intergruppenkommunikation geschieht mehrheitlich auf Italienisch (cf. ASTAT 2006a).

Diese wie eine Reihe anderer Beispiele und Überlegungen, die man an dieser Stelle anführen könnte, weisen darauf hin, dass Südtirol eine Region mit institutioneller Mehrsprachigkeit und vorwiegend einsprachig orientierten Teilgesellschaften darstellt.

Die Fakten aus der Sprachrealität Südtirols werfen viele Fragen auf, besonders im Hinblick auf die Ursachen der zum Teil unzureichenden Sprachkenntnisse bei der jugendlichen Bevölkerung.

Die letzte große Studie zu den Zweitsprachkompetenzen sowohl der deutsch- als auch der italienischsprachigen Mittel- und OberschülerInnen von Putzer und Deflorian liegt mehr als zehn Jahre zurück (Putzer/Deflorian 1997). Die Ergebnisse der Untersuchung von Vettori (2004) über die Deutschkenntnisse italienischer Mittel- und OberschülerInnen aus Bozen und Trient aus dem Jahr 2003 gab Anlass dazu, die Sprachkenntnisse der Südtiroler SchülerInnen vertiefend zu analysieren. 
Im selben Zeitraum wie die Untersuchung von Putzer und Deflorian fanden im Auftrag der Landesregierung auch die Studie "Motivation und Kontakte" (durchgeführt in den Jahren 1994-1996, Baur 1996) sowie eine Erhebung der didaktisch-methodischen Gegebenheiten des schulischen Zweitsprachunterrichts statt (Carli/Fischer/Gelmi 1997). Die Ergebnisse der Studie wurden leider nie in Buchform veröffentlicht und so einem größeren Publikum zugänglich gemacht. Außerdem wurden nie Zusammenhänge zwischen den Ergebnissen der drei Einzelstudien herausgearbeitet. Genau hier möchte die vorgestellte Studie ansetzen und zum ersten Mal versuchen, Zusammenhänge und Wechselwirkungen zwischen sprachlichen und außersprachlichen Faktoren zu ermitteln, die das Zweitsprachlernen und die Verwendung der Zweitsprache im Südtiroler Kontext beeinflussen. ${ }^{3}$ Die Untersuchung möchte damit neue Einsichten bringen sowie Hilfen für künftige sprachpolitische Entscheidungen bieten.

\subsection{Methode(n)}

Das Projekt entsteht in interdisziplinärer Zusammenarbeit zwischen Linguistinnen der Europäischen Akademie Bozen (EURAC) und (Sozial-)Psychologinnen der Universität Trient, aber auch DidaktikerInnen der Schulämter der Autonomen Provinz Bozen - Südtirol.

In der ersten Projektphase kommen qualitative Methoden zum Einsatz, nämlich Interviews mit Schlüsselfiguren aus verschiedenen Bereichen und Fokusgruppen mit SchülerInnen der 4. Klasse der Sekundarstufe II, auf die im Folgenden genauer eingegangen wird.

Die Ergebnisse aus dieser Projektphase bilden - neben dem Studium der einschlägigen Fachliteratur - eine wichtige Grundlage für den quantitativ ausgerichteten Projektteil, der aus einer Fragebogenerhebung mit SchülerInnen, Eltern und LehrerInnen und einer Sprachstandserhebung mit SchülerInnen der 4. Klasse der Sekundarstufe II besteht und Ende 2007/Anfang 2008 durchgeführt wird.

Die SchülerInnen, die an der Untersuchung teilnehmen, besuchen die 4. Klasse der Sekundarstufe II, das heißt die Klasse vor dem letzten, dem Maturajahr (Abitur), und haben ein durchschnittliches Alter von 17-18 Jahren. Zudem sind Eltern und Lehrpersonen an der Fragebogenerhebung beteiligt.

\section{Erste Ergebnisse}

\subsection{Interviewstudie}

Bereits im Jahr 2006 wurde eine erste Interviewstudie mit 16 Schlüsselfiguren, acht davon deutscher, acht italienischer Muttersprache, aus vier verschiedenen Bereichen, nämlich Politik, Medien, Schule/Universität und verschiedenen Institutionen, sowie mit unterschiedlichen beruflichen Profilen (JournalistInnen, PolitikerInnen, L2-LehrerInnen, eine Schuldirektorin, eine Erzieherin, Angestellte der Schulämter) durchgeführt. ${ }^{4}$ Ziel dieser Studie war es, Informationen für die geplante und größer angelegte Fragebogenerhebung zu gewinnen und dabei besonders soziokulturelle und psychologische Aspekte herauszuarbeiten, aber auch Impulse für die Durchführung der Fokusgruppen zu gewinnen.

\footnotetext{
3 Über viele Aspekte der sprachlichen Situation in Südtirol wurde bereits geforscht und geschrieben. An dieser Stelle mögen einige Hinweise auf weitere Arbeiten und Untersuchungen des vergangenen Jahrzehnts, die im Zuge der Vorbereitungen und Durchführung des vorgestellten Projekts berücksichtigt wurden und werden, genügen, wie z. B. ASTAT (2006a) und (2006b), Baur (2000), CENSIS (1997), Egger (2001), Lanthaler (1990) und (2006), Egger/Lanthaler (2001), Riehl (2001 und (1998).

${ }^{4}$ Die Interviews wurden von Doris Forer durchgeführt und ausgewertet. Die dargestellten Ergebnisse beruhen im Wesentlichen auf diesen ersten, bislang unveröffentlichten, Auswertungen.
} 
Die Daten wurden mithilfe semistrukturierter Leitfadeninterviews erhoben; die Gespräche wurden in Form von explorativen Experteninterviews geführt (cf. Kruse 2006: 140). Sie wurden anschließend transkribiert und inhaltlich ausgewertet.

Folgende Themenbereiche wurden angesprochen: Schule und Ausbildung, Sprachkompetenzen (L1 und L2 sowie die Rolle des Dialekts), Kontakt und Austauschmöglichkeiten, Empfindungen, Gefühle und persönliche Erfahrungen, Unterschiede zwischen den "ethnischen" oder sprachlichen Gruppen, über Politik und Medien vermittelte "Botschaften" und schließlich historische Aspekte.

Einige Ergebnisse sollen hier überblicksartig angeführt werden, die auch im Hinblick auf die anschließend vorgestellten Fokusgruppen interessant erscheinen, bei denen - im Unterschied zu den Interviews mit Erwachsenen - Jugendliche zu Wort kommen und ihre Meinungen und Ansichten kundtun.

Der Eindruck, dass die SüdtirolerInnen in parallelen Welten leben, in denen jeweils eine Sprache vorherrscht, wurde in den Interviews bestätigt. Dabei wurden als Gründe für einen erschwerten Kontakt u. a. das sprachlich getrennte Schulsystem, aber auch die territoriale Verteilung, besonders der mehrheitlich der deutschsprachigen Bevölkerung vorbehaltene ländliche Bereich, genannt.

Die Verantwortung für den sprachlichen Austausch und das Sprachenlernen wird einerseits bei der Schule gesehen, deren Aufgabe ein verbesserter Sprachunterricht sei; darüber hinaus liege der Sprachkontakt in der Verantwortung des Einzelnen und der Familien. Diese Tatsache, nämlich dass der Kontakt dem Zufall oder der Motivation Einzelner überlassen wird, gab teilweise Anlass zu Kritik. Daneben wurde besonders die Politik als Hauptverantwortlicher für den Aufbau und den Erhalt unsichtbarer Mauern genannt. Zudem scheint die Ideologie der 50er Jahre des vergangenen Jahrhunderts, die dem Motto "Je mehr wir trennen, desto besser verstehen wir uns"5 oder "Mischkultur ist Mistkultur" folgt, augenscheinlich nach wie vor im kollektiven Gedächtnis präsent zu sein, da sie in den Gesprächen immer wieder aufgetaucht ist (Interview e). Solche Grundsätze werden von einem der Interviewpartner als "latenter heimlicher Lehrplan des Landes" bezeichnet (Interview i).

Dass die Erlernung der Landessprachen Deutsch und Italienisch wichtig ist, darüber herrscht kein Zweifel. Was nun die Sprachkenntnisse an und für sich betrifft, so wird von einigen Interviewpartnern ein allgemeines Abnehmen sowohl der L1- als auch der L2-Kenntnisse konstatiert. Auffallend ist dabei vor allem der Eindruck abnehmender Deutschkenntnisse bei der deutschen Sprachgruppe. Dem gegenüber stehen beispielsweise Ergebnisse der DESIStudie, die 2003 von der deutsche Kultusministerkonferenz in Auftrag gegeben wurde und an der sich auch Südtirol beteiligt hat, und deren Ziel die Untersuchung der Deutsch- und Englischkenntnisse von SchülerInnen der 9. Schulstufe war. Die Ergebnisse dieser Studie zeigen, dass die deutschen Südtiroler SchülerInnen mit ihren Leistungen im Mittelfeld liegen und sich von denen aus Deutschland nicht in extremem Maße unterscheiden. Auch die Daten zur Selbsteinschätzung aus dem Sprachbarometer (2006a: 138-153) deuten darauf hin, dass die Muttersprachkenntnisse als positiv eingeschätzt werden.

Daneben herrscht bei den Interviewpartnern unserer Studie die Überzeugung vor, dass die L2Kenntnisse der deutschen Sprachgruppe besser seien als die der italienischen (Interviews d, i, m). Mit diesem Eindruck stimmen unter anderem die Ergebnisse des Sprachbarometers, die auf Selbsteinschätzung beruhen, oder der bereits weiter oben erwähnten, mittlerweile mehr als 10 Jahre zurückliegenden Studie von Putzer und Deflorian (1997) überein. Bei den Zweisprachigkeitsprüfungen ist es hingegen nicht möglich, die Ergebnisse den beiden

\footnotetext{
${ }^{5}$ So die Aussage des damaligen langjährigen Südtiroler Landesrats Dr. Anton Zelger.
} 
Sprachgruppen zuzuordnen; wir wissen aus dem Sprachbarometer, dass beispielsweise im Jahr 2004 38,5\% der deutsch- vs. 20,8\% der italienischsprachigen Bevölkerung im Besitz des Zweisprachigkeitsnachweises war (ASTAT 2006a: 88).

Auf der anderen Seite wird auch von einer Trendwende gesprochen, wonach die L2Kenntnisse der italienischen Sprachgruppe in den letzten Jahren zugenommen hätten (Interviews a, o). Dies wird unter anderem mit dem von Alexander Langer (1946-1995), einem grünen Politiker, der sich zeitlebens für das Zusammenleben der Sprachgruppen und die Autonomie in Südtirol engagiert hat, geprägten Begriff der "Rückverdeutschung" des Territoriums (Interview a) in Zusammenhang gebracht. Damit wird auf die Tatsache hingewiesen, dass nach den Jahren starker Italianisierung des Gebiets während der Zeit des Faschismus die deutsche Sprachgruppe in den vergangenen Jahrzehnten an Macht zurückgewonnen hat. In diesem Kontext wird in Südtirol häufig darauf hingewiesen, dass die deutsche Sprachgruppe mehr einflussreiche berufliche Posten innehabe als die italienische.

Ein wichtiger Aspekt in den Interviews bildete auch die Rolle des deutschen Dialekts, der vielfach als Haupthindernis bei der Erlernung von Deutsch als Zweitsprache gesehen wurde, eine Auffassung, die alles andere als neu ist. Der deutsche Dialekt sei in allen Domänen vorherrschend. Für die Verwendung des Standarddeutschen vonseiten der italienischsprachigen Bevölkerung fehle das entsprechende Umfeld (Interview d). Der "Dialekt" im Allgemeinen scheint bei den beiden Sprachgruppen eine unterschiedliche Relevanz zu besitzen; bei der italienischen Sprachgruppe nimmt er eine weitaus geringere Stellung ein als bei der deutschen. Dies zeigen unter anderem auch Ergebnisse aus dem Sprachbarometer (2006: 105-116), wonach italienische(r) Dialekt(e) zwar zum Teil in der Familie verwendet wird/werden, es sich aber nicht um einen lokalen Dialekt handelt, der die Zugehörigkeit zum Territorium ausdrückt, der historisch gewachsen und charakteristisch für eine Region ist.

In Bezug auf den deutschen Dialekt ${ }^{6}$ wird eher von einer Zunahme, einer Dialektrenaissance, als von einer Abnahme gesprochen, besonders bei den Jugendlichen, die ihn zunehmend auch in der schriftlichen Kommunikation (SMS, E-Mail) verwenden.

In den Gesprächen wird der Zusammenhang zwischen Dialekt und Identität thematisiert. Der Dialekt sei ein wichtiges Merkmal, das ein Individuum mit einer Gruppe teile - dies treffe besonders auf die deutsche Sprachgruppe in Südtirol zu. Der Dialekt stehe unter anderem für die Bewahrung der eigenen Identität; er wird in diesem Sinne von einigen als Schutz vor einer gefürchteten Misch-Masch-Kultur interpretiert. Auf der anderen Seite wurde der Dialekt auch als Art "Geheimsprache" (Interview i) bezeichnet, die die Funktion habe, den anderen auszuschließen.

Während der Dialekt besonders für die deutsche Sprachgruppe als positiv besetzt beschrieben wird, drückten einige Interviewpartner die Meinung aus, dass die italienische Sprachgruppe den deutschen Dialekt als wenig attraktiv, als Sprache zweiter Klasse betrachte, der an die bäuerliche Welt gebunden und nicht dazu geeignet sei, komplexere oder gar philosophische Inhalte auszudrücken (Interview o). Auf der anderen Seite wiesen einige Interviewpartner darauf hin, dass es vonseiten der italienischen Sprachgruppe auch Tendenzen gebe, sich dem Dialekt anzunähern. So würden in Südtirol eigene Dialektkurse für italienische MuttersprachlerInnen angeboten. Der Grund für die Annäherung an den Dialekt, der besonders in der Aneignung passiver Kenntnisse besteht, liege dabei weniger in der Schönheit des Dialekts, sondern in der Möglichkeit, dadurch besser in die Südtiroler Gesellschaft integriert zu werden (Interview p).

\footnotetext{
6 "Dialekt" wird hier stellvertretend für alle von der Standardsprache abweichenden Varietäten des Deutschen verwendet.
} 
"Die Südtiroler SchülerInnen und die Zweitsprache"

Zur durchgeführten Interviewstudie ließe sich noch vieles hinzufügen, das Gesagte soll aber an dieser Stelle genügen, um als Nächstes auf die Stimmen der Jugendlichen überzugehen.

\section{$2.2 \quad$ Fokusgruppen}

Insgesamt wurden sieben Fokusgruppen durchgeführt (im Folgenden zitiert als F1 bis F7), jeweils drei mit SchülerInnen von Schulen mit deutscher vs. italienischer Unterrichtssprache und eine mit SchülerInnen aus der deutschen und der italienischen Schule. Jede Gruppe bestand aus rund neun SchülerInnen der 4. Klasse allgemeinbildender und berufsbildender Oberschulen (Sekundarstufe II) aus den Städten Bozen, Brixen, Meran, Bruneck und Schlanders. Die Gruppen waren also in Bezug auf die Schulstufe und die Sprachen homogen. Ziel war es auch hier in erster Linie, Informationen und Impulse für die geplante Fragebogenerhebung zu sammeln; der Schwerpunkt der Auswertung wurde auf die Ermittlung thematisch-inhaltlicher Befunde gelegt. In jeder Fokusgruppe gab es einen Moderator sowie eine weitere Person, die für Aufnahme und Technik zuständig war, und kaum in die Gespräche eingriff. Gearbeitet wurde mit einem semistrukturierten, halboffenen Interviewleitfaden. Als Anfangstimuli wurden einerseits relativ offene, andererseits auch provokative und konfrontative Fragen und Statements verwendet. Anschließend war es wichtig, von den SchülerInnen selbst aufgeworfene Themen weiter zu verfolgen, der freien Narration und dem freien Gesprächsverlauf Platz zu lassen bzw. nicht zu sehr am Leitfaden zu "kleben" und einer Art "Leitfaden-Bürokratie" zu verfallen (cf. Reinders 2005: 239). Je nach Gesprächsverlauf griffen die ModeratorInnen mehr oder weniger steuernd ein.

Die gewählte Erhebungsmethode, die ein "interaktionsorientiertes Sinnverstehen" (cf. Kruse 2006: 160) ermöglichte, erschien für die gewählte Altersgruppe adäquat, da sie eine relativ entspannte und lockere Gesprächsatmosphäre schafft, es den Jugendlichen erleichtert, "sich unter Gleichaltrigen in einer gewohnten Sprachform und unter Rückgriff auf vertraute eigene Relevanzsysteme zu äußern" (Abel/Stuflesser in Druck), und da so "durch wechselseitige Stimulation das wesentlich Gemeinte zur Sprache" komme (Dreher/Dreher 1995: 186) und "kollektive Orientierungsmuster" (Bohnsack 2004: 374) sichtbar würden (cf. auch Abel/Stuflesser im Druck).

Drei Themenbereiche standen im Mittelpunkt der Diskussionen, nämlich a) Zweitsprachgebrauch und -kenntnisse sowie der Dialekt, b) Emotionen und Überzeugungen und schließlich c) Kontakt und Sprachgewohnheiten. Daneben kamen auch Themen wie Südtirol als zweisprachige Realität allgemein und Geschichte zur Sprache.

Die Meinungen und Ansichten zu den Sprachkompetenzen stimmen im Wesentlichen mit den Befunden anderer Studien überein (siehe dazu weiter oben). Die eigenen L2-Kompetenzen werden großteils als nicht zufrieden stellend empfunden (z. B. F1). Eine Ausnahme bilden dabei die zweisprachig aufgewachsenen SchülerInnen, deren Stimme allgemein in den bisherigen Studien über die sprachliche Situation in Südtirol sehr kurz kommt bzw. überhaupt nicht wahrgenommen wird (z. B. F4). Die als unbefriedigend empfundenen L2-Kompetenzen werden auch hier auf die demographische Verteilung, auf das Schulsystem im Allgemeinen, aber auch auf den Zweitsprachunterricht im Speziellen, und schließlich auf den Dialekt zurückgeführt. Es fehlten zudem Domänen, in denen das Hochdeutsch, das man in der Schule lerne, gesprochen werde. Dennoch wird die Hochsprache von SchülerInnen beider Sprachgruppen als wichtig für die allgemeine Verständigung bezeichnet. Einige deutschsprachige SchülerInnen sehen sie als Grundlage, auf der die Dialekte aufbauen. Besonders die italienischen MuttersprachlerInnen scheinen hinsichtlich des Deutschen eine unüberwindbares Dilemma zu empfinden, da das Hochdeutsch, das man als L2 in der Schule lerne, zwar den alleinigen Schlüssel zur Arbeitswelt darstelle bzw. den Eintritt ins Arbeitsleben ermögliche, dann jedoch der Dialekt die Sprache des täglichen Lebens in 
praktisch allen Domänen bilde (z. B. F1, F2, F4); andererseits scheinen die italienischen SchülerInnen einen Dialektunterricht in der Schule dennoch abzulehnen (F4, F5).

In Bezug auf die Verwendung der L2 werden wiederholt die Wörter "sich schämen" und "peinlich" verwendet. Viele SchülerInnen scheinen dabei den Eindruck zu haben, die zweite Sprache nur ungenügend zu beherrschen; dies führe dazu, sie auch kaum zu verwenden, mit der Folge, sie zunehmend schlechter - anstatt besser - zu beherrschen (z. B. F1, F4). Auch in einer anderen Südtiroler Interviewstudie zum Thema "Motivation und Sprachenlernen", 7 tauchte dieser Aspekt auf und es wurde die Metapher vom "Teufelskreis" verwendet, "in dem Misserfolg und Scham zirkulär zusammenhingen und einander bedingten" (Abel/Stuflesser 2006: 73). SchülerInnen italienischer Muttersprache äußerten in diesem Zusammenhang, dass sie im deutschsprachigen Ausland weniger Unsicherheiten bei der Verwendung des Deutschen hätten; das kann dadurch erklärt werden, dass dort die Erwartungen an die Deutschkompetenzen des Gesprächspartners niedriger eingeschätzt werden als in Südtirol, wo nach vielen Jahren Zweitsprachunterricht die Erwartungen entsprechend höher liegen.

Interessant ist außerdem die Tatsache, dass von einigen SchülerInnen die eigenen L1Hochdeutschkompetenzen zum Teil als negativ eingestuft wurden (z. B. F1). Das Phänomen der Asymmetrie zwischen dominanter Variante zu "anderen" Varianten bei plurizentrischen Sprachen, wie dies auch in Südtirol der Fall ist, und die damit zusammen hängenden "Unsicherheiten den Normen der eigenen Sprache gegenüber, die nicht selten zu Verleugnungshaltungen, Abwertung und Ablehnung des sprachliche Eigenen als "Dialekt" führt", und das "Nichtwissen über die Merkmale des eigenen Deutsch" (Muhr 1995: 81) ist auch aus anderen Situationen bekannt und nicht neu (cf. dazu z. B. Muhr 1995: 79-84). Zu diesen Unsicherheiten in Bezug auf die eigenen L1-Deutschkompetenzen gesellt sich die Auffassung von italienischsprachigen SchülerInnen, dass das Deutsch in Südtirol "kein gutes Deutsch" bzw. "kein richtiges Deutsch" sei (z. B. F1, F4). Diese beiden Auffassungen scheinen sich gegenseitig potenzieren, wobei - sehr vereinfachend und plakativ ausgedrückt auf der einen Seite eine Art von Minderwertigkeitsgefühl der deutschsprachigen Bevölkerung im Zusammenhang mit der eigenen Sprache das (negative) Bild der deutschen Sprache in Südtirol mitprägt, auf der anderen Seite die Beurteilung des Deutschen vonseiten der italienischsprachigen SchülerInnen dieser Auffassung quasi entspricht und zum Bild einer etwas minderwertigen deutschen Sprache in Südtirol beiträgt.

Interessant ist in diesem Zusammenhang die allgemein geteilte Überzeugung, dass Deutsch eine schwierige Sprache sei (z. B. F6). Zudem klinge Italienisch melodisch, während (Hoch)Deutsch hart anmute, eine Charakterisierung, die selbst deutschsprachige Jugendliche äußerten, die darüber hinaus zur Beschreibung des Klanges auch Wörter wie "steif", "abgehackt" und "Maschinengewehr" benutzten. Einige der deutschsprachigen SchülerInnen trennten ganz klar zwischen Hochdeutsch und Dialekt, wobei letzterer zum Teil als schöner empfunden wurde. Der Dialekt wurde als "gefühlsbetont" und "flüssiger" beschrieben, "des isch so oans, des geaht so durch" ['das ist so eines, das geht so durch'] (F1). Hochdeutsch wurde einige Male in Verbindung gebracht mit "Schule", "Noten", "Stillsitzen" und "Strammstehen" und als ein Signal für "achtung, iatz kimp eppos formals" ['Achtung, jetzt kommt etwas Formales'] (F1) gewertet. Solche Aussagen legen eine Interpretation nahe, wonach der Dialekt - im Gegensatz zum Hochdeutschen - die Sprache des Privaten sei. Dies wiederum führt zur Schlussfolgerung, dass die italienischen MuttersprachlerInnen (ohne Dialektkenntnisse) von der "deutschen" privaten Welt ausgeschlossen sind.

Neben dem etwas "schwierigen" Verhältnis zum Hochdeutschen drückten einige SchülerInnen hingegen aus, dass die Beherrschung und Verwendung des Hochdeutschen

\footnotetext{
${ }^{7}$ Die Studie wird durchgeführt im Rahmen des Projekts "Language Bridges", www.eurac.edu/Org/LanguageLaw/Multilingualism/Projects/LABS_general_de.htm.
} 
absolut normal und unproblematisch sei. Bei einigen SchülerInnen war das Bewusstsein zu bemerken, dass das Hochdeutsche in bestimmten Situationen und für bestimmte Textsorten genannt wurde hier als Beispiel etwa "einen Vortrag in Geschichte halten" oder "Nachrichten sprechen" - angemessen, der Dialekt hingegen unangebracht sei. Im Hochdeutschen einen "Südtiroler Akzent" zu haben, wird dabei von einigen SchülerInnen als "natürlich" beschrieben; "das sind einfach wir" (F 1). Dazu gesellt sich das Gefühl, dass der Dialekt als die "eigene Sprache" empfunden wird, das Hochdeutsch hingegen für einige "fast wie eine Fremdsprache" sei. Einige SchülerInnen äußerten sich zufrieden darüber, dass sie während der Gruppendiskussionen den Dialekt verwenden durften, da man sich, wie sie es beschrieben, "in der eigenen Sprache" eben besser ausdrücken könne. Der Dialekt wird auf der einen Seite als Persönlichkeitsmerkmal, als Identitätsmerkmal, als integrativer Bestandteil der eigenen Persönlichkeit gesehen (z. B. F1). Zudem ist er ein Symbol für die Zugehörigkeit $\mathrm{zu}$ einer ganzen Gruppe. Die Angst vonseiten der deutschsprachigen Jugendlichen vor "Vermischung", die einige Male genannt wurde, kann in diesem Sinne als Angst vor dem Verlust der Identität und der Identifikation bzw. Verwurzelung und dem Beheimatetsein in der eigenen Gruppe aufgefasst werden. Dem steht auf der anderen Seite ein Gefühl des Ausgeschlossenseins bzw. der "Heimatlosigkeit" bei der italienischen Sprachgruppe gegenüber. Bezeichnungen wie "Südtiroler" für die deutschsprachigen vs. "Italiener" für die italienischsprachigen BewohnerInnen des Landes können auch als Indiz für den Ausschluss der italienischen Sprachgruppe gedeutet werden.

Aufschlussreiche Ein- und Ansichten ergaben sich in diesem Zusammenhang aus der Frage, ob die SchülerInnen ihre eigenen Kinder in den jeweils anderssprachigen Kindergarten oder die anderssprachige Schule schicken würden. Hier betonten einige SchülerInnen (besonders die zweisprachig aufgewachsenen) die Vorteile, wenn Kinder entweder in einem zweisprachigen Elternhaus aufwüchsen oder eine anderssprachige Schule besuchten, da dies eine Möglichkeit sei, beide Sprachen mühelos zu lernen. Auf der anderen Seite gab es auch viele Einwände; so gab es zum Beispiel Vorbehalte, die eigenen Kinder in einen anderssprachigen Kindergarten zu schicken, "weil es wird net lai die sproch eh in die kinder beigebrocht, sondern es werden ah ondere werte irgendwie (.) italieno und daitsche sein oanfoch net gleich; do sein oanfoch untoschiede im denkn, und ih find (.), ih persönlich tat des net tian" ['denn es wird nicht nur die Sprache äh den Kindern beigebracht, sondern es werden auch andere Werte irgendwie (.) Italiener und Deutsche sind einfach nicht gleich; da sind einfach Unterschiede im Denken, und ich finde (.), ich persönlich würde das nicht tun'] (F1). Auffallend ist, dass einige SchülerInnen allerdings, was sich bei genauerem Nachfragen herausstellte, nur eine recht vage und diffuse oder überhaupt keine Vorstellung davon hatten, worum es sich bei den angesprochenen Werten, Bräuchen oder Denkweisen handle und worin sie sich konkret unterschieden (F1, F6). Es scheint die (vage) Meinung zu bestehen, dass das Aufwachsen in und mit einer einzigen Sprache und Kultur Halt und Orientierung gebe. So erwiderte eine Schülerin beispielsweise dem Vorschlag einer anderen, zweisprachig Aufgewachsenen, dass es doch das Beste wäre, wenn ein Kind die Bräuche beider Kulturen lerne, "donn woass des kind donn net, wos hinkeahrt donn im prinzip" ['dann weiß das Kind dann nicht, wo es hingehört dann im Prinzip'] (F1). Ähnliche Diskussionen und z. T. verbale Schlachten werden auch vielfach in den Medien ausgetragen. Es sei hier - stellvertretend für viele andere - an einen Vorfall im Jahr 1998 erinnert, bei dem die Kinder an einem deutschen Kindergarten bei einem Martinsumzug ein Lied mit einer Strophe auf Italienisch gesungen haben, was zu einer Flut von Zeitungsartikeln und Leserbriefen geführt hat, nicht zuletzt vom damaligen deutschen Kulturlandesrat, der darin eine schleichende Unterwanderung der Bestimmungen des Artikel 19 des Autonomiestatuts und eine mögliche Gefährdung der Entwicklung einer eigenen Identität der deutschsprachigen Kinder sah (cf. den Beitrag der Wochenzeitung "FF" Nr. 7 vom 14.02.1998: 66f.). 
Abschließend sollen noch einige Bemerkungen zum Sprachkontakt und den Sprachgewohnheiten gemacht werden: Die Intergruppenkommunikation findet in Südtirol vorwiegend auf Italienisch statt; dies zeigen nicht nur die Auswertungen der Fokusgruppen, sondern auch andere Untersuchungen. Laut Sprachbarometer (ASTAT 2006a: 131) verwendet $29,1 \%$ der deutschen Sprachgruppe in der Intergruppenkommunikation die eigene Muttersprache, 28,7\% die Sprache der des Gesprächspartners (die Tendenz, die andere Sprache zu verwenden, nimmt mit höherer Schulbildung und jüngerem Alter zu); bei 41,0\% hängt die Wahl der Sprache von der jeweiligen Situation $a b$, der Rest weiß dazu keine Antwort. Bei der italienischen Sprachgruppe hingegen verwendet 65,4\% die eigene Muttersprache, 5,4\% die Sprache der anderen Person, 25,3\% gibt an, dass dies auf die jeweilige Situation ankomme, und 4,0\% weiß es nicht.

Für den vorwiegend auf Italienisch verlaufenden Kontakt werden in den Fokusgruppen verschiedene Begründungsansätze genannt: Einmal ist man der Meinung, dass die "Deutschen" besser Italienisch könnten als umgekehrt; daher erfolge die Kommunikation zwischen den Gruppen auf Italienisch (z. B. F 6). Außerdem wurde von italienischsprachigen SchülerInnen die Ansicht geäußert, die deutschsprachigen SüdtirolerInnen schämten sich ein "tedesco pulito" ('sauberes Deutsch') zu sprechen und würden das Italienische dem Hochdeutschen vorziehen (F4); deutschsprachige SchülerInnen bestätigten, dass sie lieber Italienisch als Hochdeutsch sprächen (F6). Die Wahl des Italienischen wird von den deutschen MuttersprachlerInnen auch als Zuvorkommenheit aufgefasst, "weil man ihnen a entgegnkemmen will" ['weil man ihnen auch entgegenkommen will'] (F1). Zudem fällt auf, dass die Art des Verhaltens in der Intergruppenkommunikation als Gegebenheit, die nicht infrage zu stellen ist, als quasi "gottgegebenes Naturgesetz", als normal wahrgenommen wird, "weil des seit johre oanfoch so isch, und dass des gor nimmer aufollt" ['weil das seit Jahren einfach so ist und gar nicht mehr auffällt'] bzw. "weils eigntlich olm schon so wor" ['weil das eigentlich immer schon so war'] (F1). Die Ergebnisse des Sprachbarometers (ASTAT 2006a: 132-135) decken sich großteils mit den Äußerungen der Jugendlichen: Hier werden als Grund für die Wahl der eigenen Sprache Unsicherheit in der Verwendung der anderen Sprache, mangelnde Sprachkenntnisse und Gewohnheit genannt, außerdem der Wert, die eigene Sprache zu verwenden sowie die Auffassung, die anderen sollten sich anpassen. Gründe für die Wahl der jeweils anderen Sprache sind zum Beispiel Höflichkeit, Gewohnheit oder die Auffassung, die andere Sprache besser zu können als umgekehrt.

\section{Schlussbemerkung und Ausblick}

Im Rahmen der Interviewstudie und der Fokusgruppen wurde - neben anderen Aspekten - die Wahrnehmung der Sprachkompetenzen der SüdtirolerInnen angesprochen. Dabei ging es einerseits um die Einschätzung der eigenen Sprachkompetenzen, andererseits um die der Anderen, wobei allgemein ein recht negatives Bild entsteht. In einem weiteren Schritt gilt es nun, in größerem Rahmen zu untersuchen, wie zum einen die aktuellen Sprachkenntnisse, besonders die L2-Kenntnisse, in Südtirol eingeschätzt werden und zum anderen, welche Zielvorstellungen bezüglich des Sprachkompetenzniveaus vorherrschen. Diese Ergebnisse wiederum sind im Vergleich mit den effektiven Sprachkenntnissen zu analysieren. Dabei wird es zudem interessant sein herauszufinden, ob und in welcher Weise die Ziele und Erwartungen der Institution Schule mit den effektiven Zweitsprachkompetenzen der SchülerInnen übereinstimmen.

Im Zusammenhang mit der Erlernung der beiden Landessprachen Deutsch und Italienisch spielen Status und Prestige der Sprachen und Varietäten (dominante vs. "andere" Standardvarietäten einerseits, Dialekt andererseits) eine wichtige Rolle. Einen entscheidenden Einfluss scheint hier das Ungleichgewicht bezüglich des Stellenwerts des deutschen Dialekts bei den beiden Sprachgruppen zu spielen, der bei vielen deutschsprachigen SüdtirolerInnen 
als integrativer Bestandteil der Persönlichkeit und Teil der Identität aufgefasst wird, dem die italienischsprachigen SüdtirolerInnen hingegen teilweise ablehnend gegenüberstehen.

Zudem ist es wichtig zu untersuchen, welchen Einfluss Einstellungen gegenüber der jeweils anderen Sprache und deren SprecherInnen und das Selbstvertrauen hinsichtlich der eigenen Sprachkenntnisse auf das Sprachenlernen haben.

Des Weiteren sind die Häufigkeit und Art des Kontakts zwischen den Gruppen näher zu beleuchten. Die Äußerungen der SchülerInnen legen nahe, dass die aktuellen Spielregeln des Zusammenlebens und des Sprachgebrauchs als gegebene und unantastbare Tradition empfunden werden, die nicht weiter reflektiert werden.

Vieles spricht dafür, dass der Kontakt mit der zweiten Sprache und deren SprecherInnen großteils auf den schulischen Kontext und wenige weitere Situationen beschränkt ist, und dass nicht nur die Nachbarsprache, sondern auch der Nachbar selbst häufig fremd ist und bleibt.

Diese und andere (erste) Ergebnisse deuten darauf hin, dass es für das Erlernen der Zweitsprache wichtig ist, daraus mehr als ein Schulfach zu machen und den Jugendlichen zu vermitteln, dass die Nachbarsprache Gebrauchswert im Alltag besitzt (cf. auch Abel/Stuflesser im Druck).

\section{Literaturangaben}

Abel, Andrea/Stuflesser, Mathias (2006): "Interviewstudie zum Zusammenspiel von Überzeugungen, Erfahrungen und Sprachenlernen. Ein Werkstattbericht". In: Abel, Andrea/ Stuflesser, Mathias/Putz, Magdalena (eds.) (2006): Mehrsprachigkeit in Europa. Erfahrungen, Bedürfnisse, Gute Praxis. Bozen: 65-76.

Abel, Andrea/Stuflesser, Mathias (im Druck): Language-Bridges-Interviewstudie zum Zusammenspiel von Überzeugungen, Erfahrungen und Sprachenlernen: Methodenfragen und Ergebnisauswertung. Erscheint in der Reihe Sprachenlernen Konkret! Hohengehren.

ASTAT - Landesinstitut für Statistik - Autonome Provinz Bozen-Südtirol (ed.) (2006a): Südtiroler Sprachbarometer 2004. Bozen.

ASTAT - Landesinstitut für Statistik - Autonome Provinz Bozen-Südtirol (ed.) (2006b): Zwei- und Dreisprachigkeitsprüfungen 2005. Bozen.

Baur, Siegried (1996): Relazione complessiava sulla ricerca "Motivazione e contatti". Condizioni di base dell'apprendimento della seconda lingua in Alto Adige. Bolzano. Unveröff. Studie.

Baur, Siegfried (2000): Die Tücken der Nähe. Kommunikation und Kooperation in Mehrheits/Minderheitssituationen. Meran.

Bohnsack, Ralf et al. (eds.) (2003): Hauptbegriffe Qualitativer Sozialforschung. Opladen.

Carli, Augusto/Fischer, Maria Luise/Gelmi, Rita (eds.) (1997): Condizioni didatticometodologiche dell'insegnamento della lingua seconda. Comunicazione in lingua seconda in contesto scolastico. Bozen. Unveröff. Studie.

CENSIS (Centro studi nazionali investimenti sociali)/Autonome Provinz Bozen-Südtirol (eds.) (1997): Identität und Mobilität der drei Sprachgruppen. Abschließender Bericht. Rom.

Dreher, Michael/Dreher, Eva (1995): "Gruppendiskussionsverfahren". In: Flick, Uwe/von Kardoff, Ernst et al. (Hrsgg.): Handbuch qualitative Sozialforschung. Grundlagen, Konzepte, Methoden und Anwendungen. Weinheim: 186-188.

Egger, Kurt (2001): Sprachlandschaft im Wandel. Bozen.

Egger, Kurt/Lanthaler, Franz (2001): Die deutsche Sprache in Südtirol. Einheitssprache und regionale Vielfalt. Wien/Bozen.

Kruse, Jan (2006): Einführung in die Qualitative Interviewforschung. Freiburg. Unveröff. Textsammlung. 
Lanthaler, Franz (1990): "Dialekt und Zweisprachigkeit". In: Lanthaler, Franz (ed.): Mehr als eine Sprache. Meran: 57-81.

Lanthaler, Franz (2006): "Die Vielschichtigkeit des Deutschen in Südtirol - und wie wir damit umgehen". In: Abel, Andrea/Stuflesser, Mathias/Putz, Magdalena (eds.): Mehrsprachigkeit in Europa. Erfahrungen, Bedürfnisse, Gute Praxis. Bozen: 371-380.

Muhr, Rudolf (1995): "Zur Sprachsituation in Österrich und zum Begriff 'Standardsprache' in plurizentrischen Sprachen. Sprache und Identität in Österreich". In: Muhr, Rudolf/Schrodt, Richard/Wiesinger, Peter (eds.): Österreichisches Deutsch. Linguistische, sozialpsychologische und sprachpolitische Aspekte einer nationalen Variante des Deutschen. Wien: 75120.

Putzer, Oskar/Deflorian, Floriano (1997): Ricerca sulle competenze in seconda lingua nelle scuole della Provincia di Bolzano. Unveröff. Studie.

Reinders, Heinz (2005): Qualitative Interviews mit Jugendlichen führen. Ein Leitfaden. München.

Riehl, Claudia Maria (1998): "Schriftsprachliche Kompetenz und Zweisprachigkeit: Der Fall Südtirol". In: Werlen, Iwar (ed.): Mehrsprachigkeit im Alpenraum. Aarau/Frankfurt/ Salzburg:175-195.

Riehl, Claudia Maria (2001): Schreiben, Text und Mehrsprachigkeit. Zur Textproduktion in mehrsprachigen Gesellschaften am Beispiel der deutschsprachigen Minderheiten in Südtirol und Ostbelgien. Tübingen.

Vettori, Chiara (2004): La competenza del tedesco degli studenti italofoni di scuola media inferiore e superiore di Bolzano e Trento: confronto e valutazione. Modena. Dissertation, Università di Modena. 